\title{
ARTICLE OPEN \\ USP7 deubiquitinates and stabilizes NOTCH1 in T-cell acute lymphoblastic leukemia
}

\author{
Huizhuang Shan ${ }^{1}$, Xiangyun $\mathrm{Li}^{1}$, Xinhua Xiao ${ }^{2}$, Yuting Dai ${ }^{2}$, Jinyan Huang ${ }^{2}$, Junjun Song ${ }^{3}$, Meng Liu' ${ }^{1}$ Li Yang ${ }^{1}$, Hu Lei ${ }^{1}$, Yin Tong ${ }^{4}$,
} Li Zhou' ${ }^{2}$, Hanzhang $\mathrm{Xu}^{1}$ and Yingli Wu (iD) ${ }^{1}$

T-cell acute lymphoblastic leukemia (T-ALL) is a highly aggressive leukemia that is primarily caused by aberrant activation of the NOTCH1 signaling pathway. Recent studies have revealed that posttranslational modifications, such as ubiquitination, regulate NOTCH1 stability, activity, and localization. However, the specific deubiquitinase that affects NOTCH1 protein stability remains unestablished. Here, we report that ubiquitin-specific protease 7 (USP7) can stabilize NOTCH1. USP7 deubiquitinated NOTCH1 in vivo and in vitro, whereas knockdown of USP7 increased the ubiquitination of NOTCH1. USP7 interacted with NOTCH1 protein in T-ALL cells, and the MATH and UBL domains of USP7 were responsible for this interaction. Depletion of USP7 significantly suppressed the proliferation of T-ALL cells in vitro and in vivo, accompanied by downregulation of the NOTCH1 protein level. Similarly, pharmacologic inhibition of USP7 led to apoptosis of T-ALL cells. More importantly, we found that USP7 was significantly upregulated in human T-ALL cell lines and patient samples, and a USP7 inhibitor exhibited cell cytotoxicity toward primary T-ALL cells, indicating the clinical relevance of these findings. Overall, our results demonstrate that USP7 is a novel deubiquitinase that stabilizes NOTCH1. Therefore, USP7 may be a promising therapeutic target in the currently incurable T-ALL.

Signal Transduction and Targeted Therapy (2018)3:29; https://doi.org/10.1038/s41392-018-0028-3

\section{INTRODUCTION}

The NOTCH1 receptor is a transmembrane protein that serves as a ligand-activated transcription factor that regulates a great diversity of cellular events, including cell proliferation, survival, metastasis, and differentiation. ${ }^{1}$ Upon ligand binding, NOTCH1 is initially cleaved by an ADAM metalloprotease in tandem with the $\gamma$-secretase complex, which releases the intracellular domain of NOTCH1 (ICN1). Then, ICN1 translocates into the nucleus and activates NOTCH1 target genes, such as c-Myc, Hes1, and Dtx $1 .^{2-5}$ Activating mutations in NOTCH1 that induce ligand-independent activation of the receptor or an increase in the stability of ICN1 are found in more than $60 \%$ of human T-cell acute lymphoblastic leukemia (T-ALL) cases. T-ALL is one of the most aggressive leukemias and has a poor prognosis. ${ }^{6-11}$ A tremendous amount of research has focused on the oncogenic mechanisms by which NOTCH1 enhances leukemogenesis via downstream genes or interaction with other important signaling pathways, such as NF$\mathrm{KB}$ and PI3K-AKT-mTOR pathways. ${ }^{12,13}$ However, the upstream mechanisms sustaining aberrant NOTCH1 signaling activities are incompletely understood, especially NOTCH1 protein turnover.

It is known that the ubiquitin-proteasome system and lysosome pathway participate in the regulation of NOTCH1 turnover. For instance, the E3 ubiquitin ligases F-box and WD repeat domaincontaining 7 (FBW7) and C-terminus of Hsc70-interacting protein (CHIP) mediate polyubiquitination of $\mathrm{NOTCH} 1$ for proteasome degradation. ${ }^{14,15}$ NOTCH1 interacts with and is monoubiquitinated by the E3 ubiquitin ligase $\mathrm{c}-\mathrm{Cbl}$ and is subsequently degraded by lysosomes. ${ }^{16}$ Ubiquitination is a reversible process, and removal of ubiquitin from proteins is mediated by deubiquitinases (DUBs), the number of which in mammalian cells is $\sim 100$. More than the half of DUBs belong to the ubiquitin-specific protease (USP) subfamily. ${ }^{17}$ To date, elF3f has been reported to function as a deubiquitinase and to regulate the activation of NOTCH1. ${ }^{18}$ However, the deubiquitinase that modulates the stability of NOTCH1 protein remains unknown.

USP7 is the most widely studied DUB and is well known as herpes-associated USP (HAUSP). ${ }^{19}$ Through its deubiquitination activity, USP7 can influence the localization, activation, and stability of its substrates. For example, USP7 changes the localization of monoubiquitinated FOXO4 and PTEN through removal of the single ubiquitin molecule $\mathrm{e}^{20-22}$ and can regulate the stability of p53, MDM2, N-MYC, TRIP12, FOXP3, ASXL1, UHRF1, PHF8, and DNMT1. ${ }^{23-30}$ Many of the preceding factors are critical in cancer development, epigenetic control, cell signaling, DNA damage repair, and immune responses. Notably, overexpression of USP7 has been detected in multiple myeloma, neuroblastoma, hepatocellular carcinoma, prostate cancer, breast cancer, and ovarian cancer, in which inhibition of USP7 suppresses proliferation and induces death of cancer cells independently of their p53 status. Considering the crucial role of USP7 in cancer

\footnotetext{
${ }^{1}$ Hongqiao International Institute of Medicine, Shanghai Tongren Hospital/Faculty of Basic Medicine, Chemical Biology Division of Shanghai Universities E-Institutes, Key Laboratory of Cell Differentiation and Apoptosis of the Chinese Ministry of Education, Shanghai Jiao Tong University School of Medicine, Shanghai 200025 , China; ${ }^{2}$ Department of Hematology, Rui-Jin Hospital, Shanghai Jiao Tong University School of Medicine, No.197, Ruijin Er Road, Shanghai, China; ${ }^{3}$ Shanghai University of Medicine \& Health Sciences, No.279, Zhouzhu Road, Shanghai, China and ${ }^{4}$ Department of Hematology, Shanghai First People's Hospital, Shanghai Jiao Tong University School of Medicine, Shanghai 200080, China

Correspondence: Yingli Wu (wuyingli@shsmu.edu.cn)

These authors contributed equally: Huizhuang Shan, Xiangyun Li and Xinhua Xiao
}

Received: 26 June 2018 Revised: 29 August 2018 Accepted: 12 September 2018

Published online: 26 October 2018 
development, much attention has been paid to developing USP7 inhibitors for cancer therapy. ${ }^{31-35}$

In this study, we confirmed that USP7 is a novel deubiquitinase that reverses NOTCH1 polyubiquitination and stabilizes NOTCH1 protein. Inhibition of USP7 led to NOTCH1 degradation and suppressed T-ALL cell proliferation in vitro and in vivo. Our data suggest that targeting the USP7/NOTCH1 axis is a novel strategy to combat T-ALL and other NOTCH1-related malignancies.

\section{MATERIALS AND METHODS}

Cell culture, patient samples, and transfection

The human T-ALL cell lines JURKAT and MOLT-4 and human embryonic kidney (HEK293T) cells were purchased from the American Type Culture Collection (ATCC, Manassas, VA, USA). CUTLL1 cells were a gift from Dr. Qingyi Tong (Huazhong University of Science and Technology, Wuhan, China); CCRFCEM, KOPT-K1, SIL-ALL, HPB-ALL, DND41, and LOUCY cell lines were kindly provided by Dr. Xinhua Xiao (Shanghai Jiao Tong University School of Medicine, Shanghai, China). T-ALL cell lines were cultured in RPMI-1640 medium with $2 \mathrm{mM}$ L-glutamine (Gibco Invitrogen Corp., Grand Island, NY, USA) supplemented with 10\% fetal bovine serum (FBS; Gibco) and 1\% penicillin/ streptomycin (Gibco). HEK293T cells were cultured in Dulbecco's modified Eagle's medium (DMEM; HyClone, Logan, UT, USA) containing $10 \%$ FBS and $1 \%$ penicillin/streptomycin. Peripheral blood mononuclear cells (PBMCs) were isolated from normal healthy donors or T-ALL patient samples provided by the Department of Hematology, Rui-Jin Hospital, Shanghai Jiao Tong University School of Medicine, Shanghai, China. Studies were carried out in accordance with guidelines approved by the Clinical Investigational Reviewing Board of the Shanghai Jiao Tong University School of Medicine. The cells listed above were maintained at $37^{\circ} \mathrm{C}$ in a humidified incubator with 95\% air and $5 \% \mathrm{CO}_{2}$. HEK293T cells were transfected with plasmids using polyethyleneimine (PEl; Polysciences, Warrington, PA, USA) according to the manufacturer's instructions.

Plasmids, antibodies, and reagents

DUB plasmids and HA-ubiquitin were purchased from Addgene (Cambridge, MA, USA). USP7 ${ }^{\text {WT }}$ (USP7 wild-type) and USP7 $7^{\text {C223S }}$ (USP7 catalytic mutant) were cloned into a pFLAG-CMV-4 vector. GFP-tagged USP7 constructs (WT or mutants) were kindly provided by Prof. Jing Liu (Central South University, Changsha, China). Additionally, pcDNA3-Myc-ICN1 and pcDNA3-FLAG-ICN1 were obtained from Prof. Hudan Liu (Wuhan University, Wuhan, China).

The following antibodies were used in this study: anti-cleaved NOTCH1 (Val1744) and anti- $\beta$-Actin (CST, Danves, MA, USA); antiUSP7 (Bethyl Laboratories, Montgomery, TX, USA); anti-NOTCH1 and anti-GFP (Santa Cruz, Dallas, TX, USA); anti-HA and anti-Myc epitope tag (MBL, Nagoya, Japan); anti-FLAG (M2) (Sigma-Aldrich, Louis, MO, USA); and anti-HRP-conjugated secondary antibody (Millipore, Bedford, MA, USA). All antibodies were diluted according to the manufacturer's recommendations.

The USP7 inhibitor P22077 [1-(5-((2,4-difluorophenyl)thio)-4nitrothiophen-2-yl) ethanone] was obtained from EMD Millipore (EMD Millipore, Billerica, MA, USA). MG132 and cycloheximide (CHX) were purchased from Sigma-Aldrich.

Retroviral transduction and infection

shRNA sequences were inserted into a PSIREN-RetroQ Vector (Clontech Laboratories, Inc., CA, USA), and then, HEK293T cells were co-transfected with the construct and packaging plasmids (pGag-Pol and pVSV-g). At $48 \mathrm{~h}$ posttransfection, the supernatants were collected and filtered using a $0.45-\mu \mathrm{m}$ cellulose acetate filter. Further, the JURKAT and MOLT-4 cells were incubated in culture medium with supernatants containing virus particles and supplemented with $8 \mu \mathrm{g} / \mathrm{mL}$ polybrene (Sigma-Aldrich) for $8 \mathrm{~h}$, followed by replacement with fresh medium. At $48 \mathrm{~h}$ postinfection, puromycin $(2 \mu \mathrm{g} / \mathrm{mL}$; Calbiochem, Merck KGaA, Darmstadt, Germany) was added to screen the cells. Control shRNA was synthesized by Sangon Biotech (Shanghai, China), and the following USP7 shRNA-1 and USP7 shRNA-2 sequences were used: USP7 shRNA-1, 5'-TGCGAAATCTGCCATGGAA-3'; USP7 shRNA-2, 5'-CTCAGAACCCTGTGATCAA-3'.

RNA extraction and quantitative real-time PCR

Total RNA was extracted with TRlzol reagent (Invitrogen), and CDNA was synthesized using a reverse transcriptase kit (Thermo Scientific, Waltham, MA, USA), followed by qRT-PCR analysis using SYBR-Green qPCR master mix (Thermo Scientific) and an ABI PRISM 7900 system (Thermo Scientific). The primer sequences were as follows: NOTCH1 forward 5'-CCGCAGTTGTGCTCCTGAA-3' and NOTCH1 reverse $5^{\prime}$-ACCTTGGCGGTCTCGTAGCT-3'; $\beta$-actin forward $5^{\prime}$-CATCCTCACCCTGAAGTACCC- $3^{\prime}$ and $\beta$-actin reverse $5^{\prime}$ AGCCTGGATAGCAACGTACATG-3'.

\section{Immunoprecipitation and western blotting}

For immunoprecipitation assays, cells were lysed in lysis buffer (1\% Nonidet P-40; $50 \mathrm{mM}$ Tris- $\mathrm{HCl}, \mathrm{pH} 7.4 ; 150 \mathrm{mM} \mathrm{NaCl} ; 5 \mathrm{mM}$ EDTA; $0.02 \%$ SDS) supplemented with protease inhibitors (Roche Applied Science) on ice for $30 \mathrm{~min}$. After centrifugation at $4{ }^{\circ} \mathrm{C}$ and $12,000 \mathrm{rpm}$ for $20 \mathrm{~min}$, the supernatants were incubated with the indicated primary antibodies at $4{ }^{\circ} \mathrm{C}$ overnight. Protein A/G Plus agarose beads (Santa Cruz) were then added for $3 \mathrm{~h}$. Next, the beads were washed with the lysis buffer three times. Finally, the bound proteins were dissolved in $2 \times$ SDS-PAGE loading buffer and analyzed by western blotting. For in vivo deubiquitination assays, cells were transfected with the aforementioned plasmids for $48 \mathrm{~h}$. $4 \mathrm{~h}$ before the collection of cell lysates, $10 \mu \mathrm{M}$ MG132 was added. Cell lysates were then subjected to immunoprecipitation experiments as described above.

For western blotting, cells were collected and lysed with $1 \times$ SDS-PAGE sample buffer. Approximately $30 \mu \mathrm{g}$ of total protein extracts was resolved on $8-15 \%$ SDS-PAGE gels (8-15\%), electrophoresed, and transferred to a nitrocellulose membrane (Bio-Rad, Hercules, CA, USA). To ensure equal protein loading, the blots were stained with $0.2 \%$ Ponceau $\mathrm{S}$ red. After being blocked with $5 \%$ nonfat milk in phosphate-buffered saline (PBS), the membranes were incubated with the antibodies specified above at $4{ }^{\circ} \mathrm{C}$ overnight, followed by incubation with an HRP-conjugated secondary antibody for $1 \mathrm{~h}$ at room temperature (RT). Finally, the signals were measured by chemiluminescence (ECL, Amersham, Little Chalfont, UK).

In vitro deubiquitination assay

For the in vitro deubiquitination assay, Myc-tagged ICN1 was coexpressed with HA-tagged ubiquitin in HEK293T cells and purified using an anti-Myc antibody and Protein A/G Plus agarose beads under denaturing conditions (50 mM Tris- $\mathrm{HCl}, \mathrm{pH} 8.0 ; 50 \mathrm{mM}$ $\mathrm{NaCl} ; 10 \mathrm{mM}$ DTT; $1 \mathrm{mM}$ EDTA and 5\% glycerol). Next, ubiquitinated-ICN1 proteins were incubated with purified USP7 protein (SinoBiological Inc., Beijing, China) in deubiquitination buffer at $37^{\circ} \mathrm{C}$ for $2 \mathrm{~h}$. This reaction was terminated by boiling in $5 \times$ SDS-PAGE sample buffer for $10 \mathrm{~min}$. Then, the samples were resolved on $8 \%$ SDS-PAGE gels, followed by western blotting analysis.

\section{GST pull-down assay}

Bacterial-expressed GST and commercially available GST-USP7 were bound to glutathione-Sepharose 4B beads (GE Healthcare, Sunnyvale, CA, USA). FLAG-tagged ICN1 was expressed in HEK293T cells. Cells were lysed and immunoprecipitated with anti-FLAG M2 beads (Sigma-Aldrich), and then, purified ICN1 protein was eluted using a $3 \times$ FLAG peptide (Sigma-Aldrich). 
a

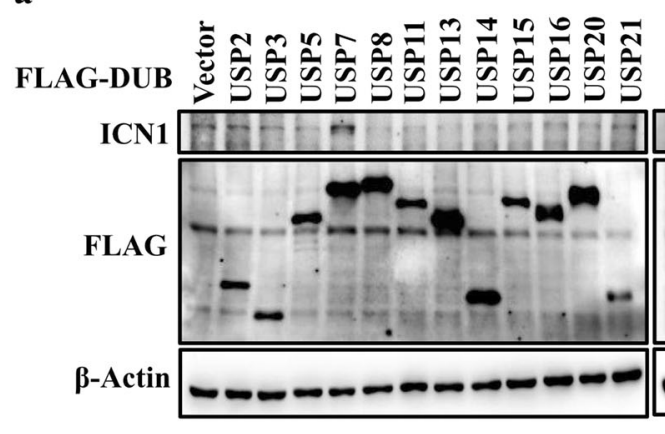

b

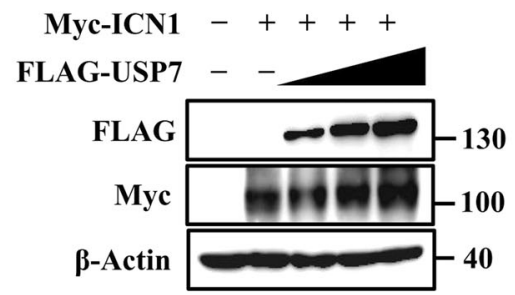

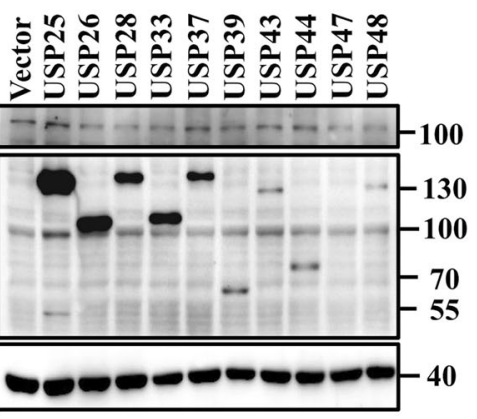
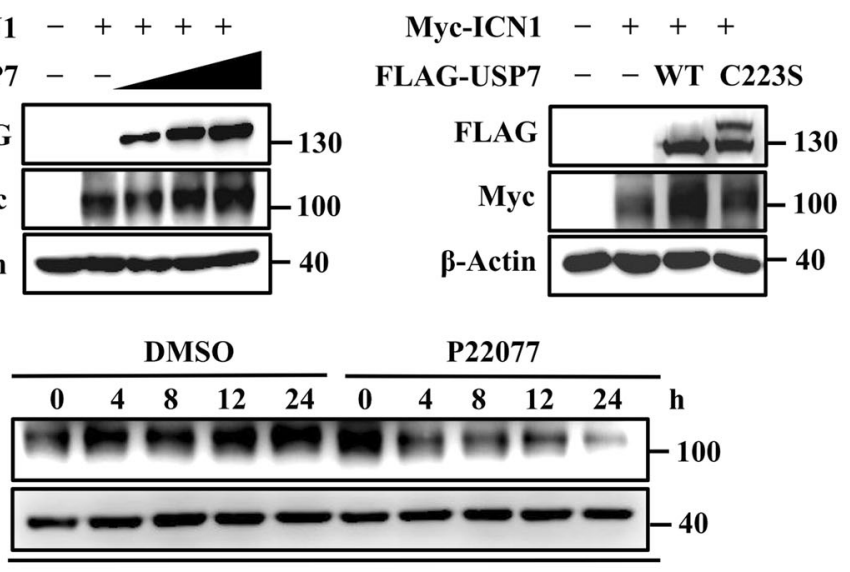

CHX pretreatment d

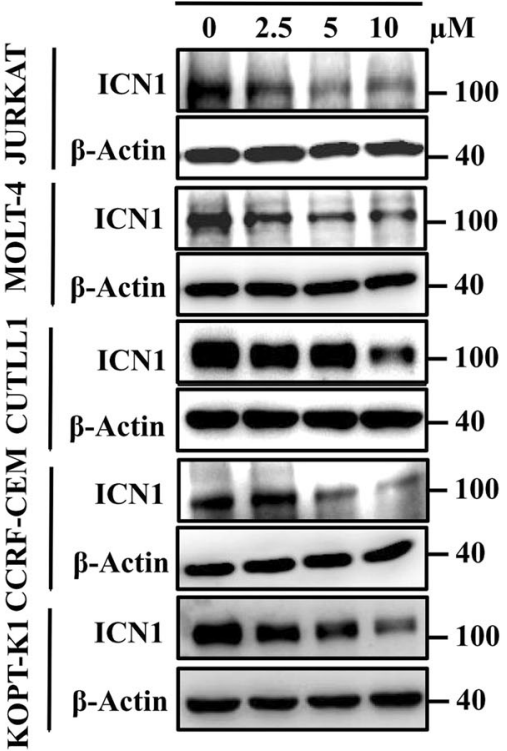

e $\mathbf{P 2 2 0 7 7}-+\quad+\quad+$

MG132 - -++

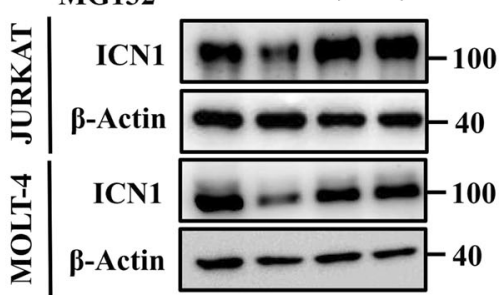

Fig. 1 The deubiquitinase USP7 stabilizes ICN1. a HEK293T cells were transfected with FLAG-tagged USPs. Cell lysates were subjected to western blotting with anti-FLAG and anti-ICN1 antibodies. b HEK293T cells were co-transfected with plasmids encoding Myc-tagged ICN1 and FLAG-tagged USP7 (increasing amounts) or its inactive C223S mutant. Cell lysates were subjected to western blotting with anti-FLAG and antiMyc antibodies. c HEK293T cells were transfected with Myc-tagged ICN1 and pretreated with CHX $(100 \mu \mathrm{g} / \mathrm{mL})$ for $2 \mathrm{~h}$. The cells were harvested at the indicated time points upon DMSO or P22077 $(10 \mu \mathrm{M})$ treatment followed by western blotting with an anti-Myc antibody. d The indicated T-ALL cell lines were treated with increasing concentrations of P22077 for $6 \mathrm{~h}$, and the ICN1 protein levels were measured by western blotting. e JURKAT and MOLT-4 cells were treated with P22077 $(10 \mu \mathrm{M})$ in the presence or absence of MG132 $(5 \mu \mathrm{M})$ for $6 \mathrm{~h}$; the indicated proteins were then examined by western blotting

FLAG-tagged ICN1 protein was incubated with GST or GST-USP7 protein in buffer $(10 \mathrm{mM}$ Tris- $\mathrm{HCl}, \mathrm{pH} 8.0 ; 100 \mathrm{mM} \mathrm{NaCl} ; 1 \mathrm{mM}$ EDTA) at $4{ }^{\circ} \mathrm{C}$ for $4 \mathrm{~h}$. The beads were washed three times and boiled in $2 \times$ SDS-PAGE loading buffer, followed by western blotting analysis.

Analysis of apoptosis via flow cytometry

Apoptosis in T-ALL cell lines was evaluated using Annexin V-APC and propidium iodide (PI; BD Pharmingen, San Jose, USA) staining. Briefly, cells were treated with different concentrations of P22077 for $48 \mathrm{~h}$. The apoptotic cells were then washed with PBS thrice and stained with Annexin V-APC and PI according to the manufacturer's protocol. Early apoptotic cells (Annexin V-positive, PInegative) and late apoptotic cells (Annexin V-positive, PI-positive) were then determined by flow cytometry (BD Biosciences, San Diego, CA, USA), and the results were analyzed using FlowJo 7.6 software (Tree Star, Ashland, OR, USA).

Cell proliferation analysis

Cell proliferation was determined using a Cell Counting Kit-8 assay kit (Dojindo Laboratories, Kumamoto, Japan). T-ALL cells were seeded into 96 -well plates $\left(4 \times 10^{3}\right.$ cells/well) and then treated with different concentrations of P22077. After incubation at $37^{\circ} \mathrm{C}$ for a different number of days, $10 \mu \mathrm{L}$ of CCK-8 reagent was added to each well. Further, incubation for another $4 \mathrm{~h}$ was performed, and the optical density (OD) at $450 \mathrm{~nm}$ was determined using a Synergy H4 Hybrid Microplate Reader (Synergy H4, Biotek, Winooski, VT, USA).

Immunofluorescence assay

Cells were fixed with $4 \%$ paraformaldehyde, permeabilized with $0.5 \%$ Triton X-100 for $15 \mathrm{~min}$ at RT, and blocked with $2 \%$ bovine serum albumin in PBS for $2 \mathrm{~h}$ at RT. The cells were next incubated with the indicated NOTCH1 (dilution 1:50; Santa Cruz) and USP7 (dilution 1:100; Bethyl Laboratories) primary antibodies overnight at $4{ }^{\circ} \mathrm{C}$, followed by incubation with a TRITC-conjugated or FITCconjugated secondary antibody (dilution 1:200; Invitrogen) for $2 \mathrm{~h}$ at RT. The cell nuclei were counterstained with 4,6-diamidino-2phenylindole (DAPI; Molecular Probes, Eugene, OR, USA). Confocal imaging was performed using a laser confocal microscope (Nikon, Nagoya, Japan).

Gene expression analysis of USP7 using databases

The USP7 transcript expression was assessed in human cell lines using the Cancer Cell Line Encyclopedia (CCLE). ${ }^{36}$ The "Haferlach Leukemia" and "Andersson Leukemia" datasets from the 
Oncomine database (http://www.oncomine.org/) ${ }^{37}$ were employed to analyze the differential expression of USP7 in T-ALL and normal marrow or peripheral blood cells.

RNA-seq data of 130 T-ALL samples were downloaded from the Chinese Genotype-Phenotype Archive of Hematology (http:// bioinfo.rjh.com.cn/cga/) under accession no. CGAS00000000002. Mutation data were obtained from a supplementary table in ref. ${ }^{38}$ Samples with USP7 mutation $(n=12)$ were not taken into account in the downstream analysis. RNA-seq data were aligned against the human reference genome hg19 using STAR (Version 2.5.3a). ${ }^{39}$ HTSeq (Version 0.9.1) was used to generate a table of the counts from the STAR output. ${ }^{40}$ All read counts were normalized and applied with variance-stabilizing transformation in the DESeq2 package. $^{41}$

\section{T-ALL xenografts}

Female NOD/SCID/IL2Ry-null (NSG) mice aged six to 8 weeks were kept under pathogen-free conditions according to the Shanghai Medical Experimental Animal Care guidelines. Human JURKAT cells transduced with retroviruses encoding pSIRENControl shRNA or pSIREN-USP7 shRNA-2 were intravenously injected into NSG mice $\left(2.5 \times 10^{6}\right.$ cells in $100 \mu \mathrm{L}$ of PBS). Disease progression was monitored weekly by Wright's staining of peripheral blood. After 3 weeks, the mice were killed. Tissue samples were fixed in formaldehyde and further processed for hematoxylin and eosin (H\&E) staining. The animal protocols were approved by the Institutional Animal Care and Use Committee of Shanghai Jiao Tong University School of Medicine.

\section{Statistical analysis}

All graphs were obtained using GraphPad Prism 5 software (GraphPad Software Inc., La Jolla, CA, USA). The data were obtained from three independent experiments and are presented as the mean \pm standard deviation (SD). Student's $t$-test was used to determine the significance of differences between groups; $p<$ 0.05 was considered to indicate statistical significance $\left({ }^{*} p<0.05\right.$; $\left.p<0.01 ;{ }^{* * *} p<0.001\right)$.

\section{RESULTS}

USP7 maintains ICN1 stability

USPs are the largest subfamily of DUBs. To explore which DUB is responsible for ICN1 stability, a total of 22 USPs were overexpressed in HEK293T cells, and the protein level of endogenous ICN1 was analyzed. This screening revealed that USP7 dramatically upregulated ICN1 levels (Fig. 1a). Then, to confirm this result, HEK293T cells were co-transfected with ICN1 and different doses of USP7 (USP7 ${ }^{\mathrm{WT}}$ ) plasmids, and as expected, the overexpression of USP7 remarkably increased ICN1 levels in a dose-dependent manner (Fig. 1b, left panel). Moreover, regulation of the ICN1 protein level by USP7 was dependent on its DUB activity, as shown by the inability of a catalytically inactive USP7 mutant (USP7 ${ }^{\text {C223S }}$ ) to upregulate ICN1 (Fig. 1b, right panel). Consistent with the above results, P22077, ${ }^{42}$ a USP7 inhibitor, significantly shortened the half-life of exogenous ICN1 in HEK293T cells (Fig. 1C).

Considering that NOTCH1 plays a critical role in T-ALL, we next examined how inhibition of USP7 would influence NOTCH1. a

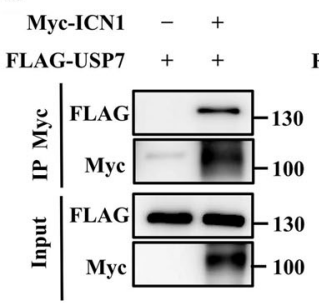

c

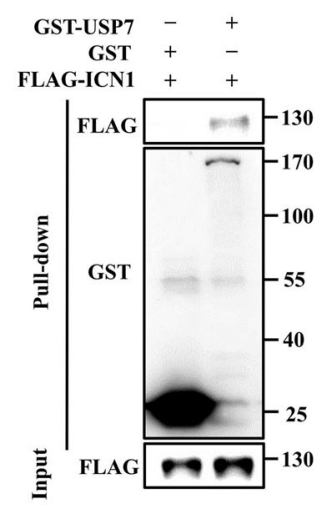

b

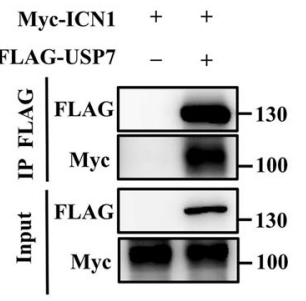

d

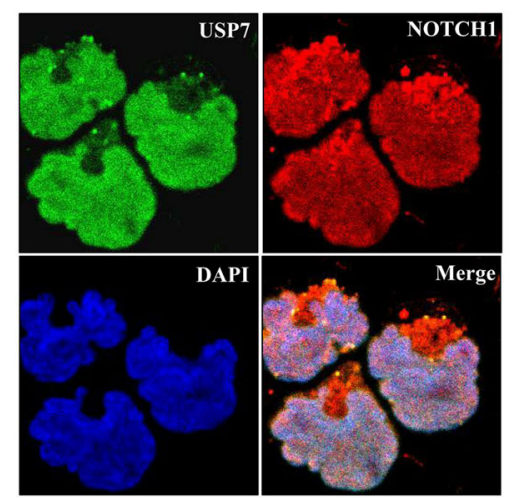

e

\begin{tabular}{|c|c|c|c|c|c|}
\hline & 20 & 560 & 801 & 1102 & Binding \\
\hline USP7 FL & MATH & CAT & UBL & & + \\
\hline 208-1102 & & $\mathrm{CAT}$ & UBL & & + \\
\hline 1-801 & MATH & $\mathrm{CAT}$ & UBL(1-2) & & + \\
\hline 208-801 & & CAT & UBL(1-2) & & + \\
\hline $1-560$ & MATH & $\mathrm{CAT}$ & & & + \\
\hline $208-560$ & & CAT & & & - \\
\hline
\end{tabular}

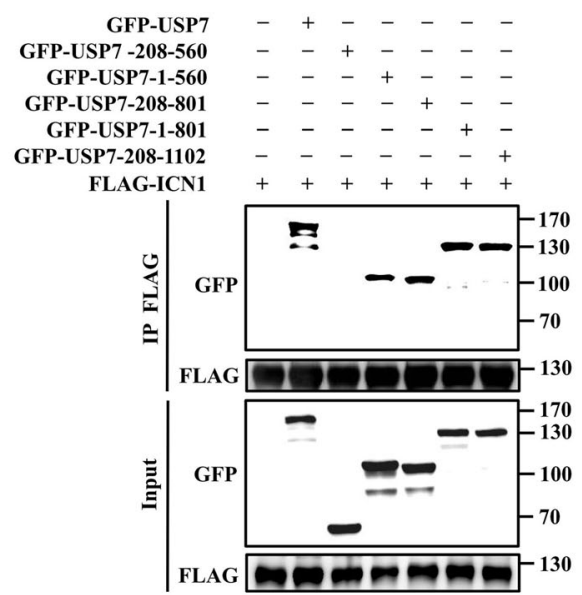

Fig. 2 USP7 interacts with ICN1. a HEK293T cells were transfected with plasmids encoding FLAG-tagged USP7 and/or Myc-tagged ICN1. Cell extracts were prepared and immunoprecipitated with anti-FLAG or anti-Myc antibodies. The protein interactions were analyzed by western blotting. b Whole-cell lysates from JURKAT and MOLT-4 cells were subjected to immunoprecipitation with a control IgG or an anti-ICN1 antibody. The immunoprecipitates were detected by western blotting. The input represented $~ 5 \%$ of the total protein extract used for immunoprecipitation. c The direct interaction between USP7 and ICN1 was detected using a GST pull-down assay, and the indicated proteins were examined by western blotting. d USP7 was co-localized with NOTCH1. CUTLL1 cells were fixed and immunostained with anti-USP7 (green) and anti-NOTCH1 (red) antibodies. The cell nuclei were counterstained with DAPI (blue). e Mapping of the ICN1-interacting domain in the USP7 protein. Top panel, a schematic representation of various USP7 truncated mutants. Bottom panel, HEK293T cells were co-transfected with constructs encoding FLAG-tagged ICN1 and GFP-tagged USP7 or truncated mutants. FLAG-tagged ICN1 proteins were immunoprecipitated with an anti-FLAG antibody, and the presence of USP7 protein and truncated mutants was examined by western blotting using an anti-GFP antibody 
a

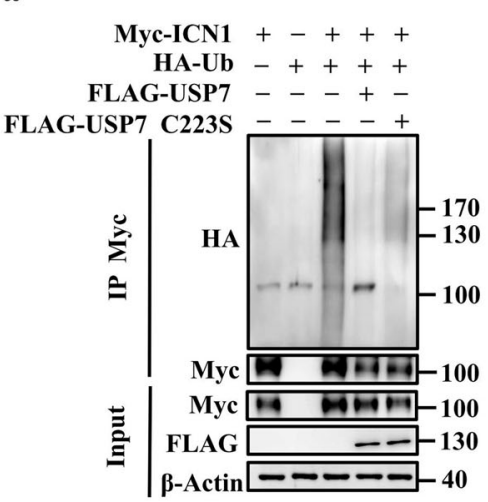

d

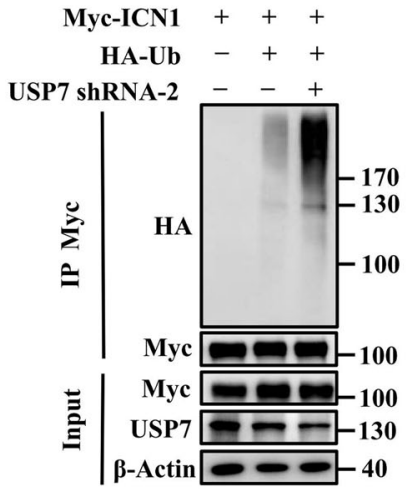

b

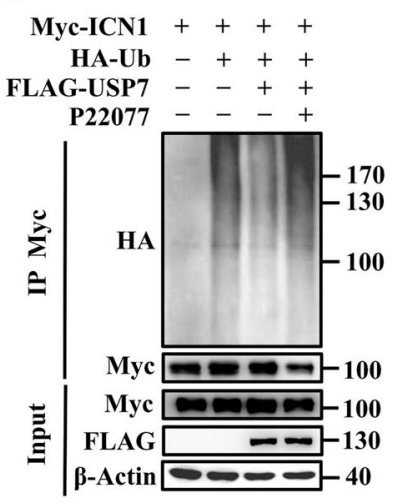

e

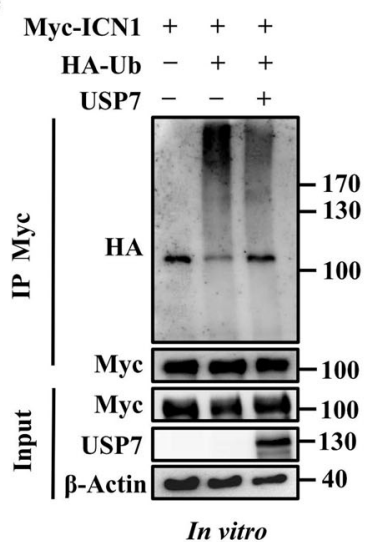

c

\begin{tabular}{r|cccccc} 
& 1 & 208 & & 560 & 801 & 1102 \\
USP7 FL & MATH & CAT & UBL & \\
\cline { 2 - 5 } USP7/AMATH & & CAT & UBL \\
\hline
\end{tabular}

USP7/AUBL

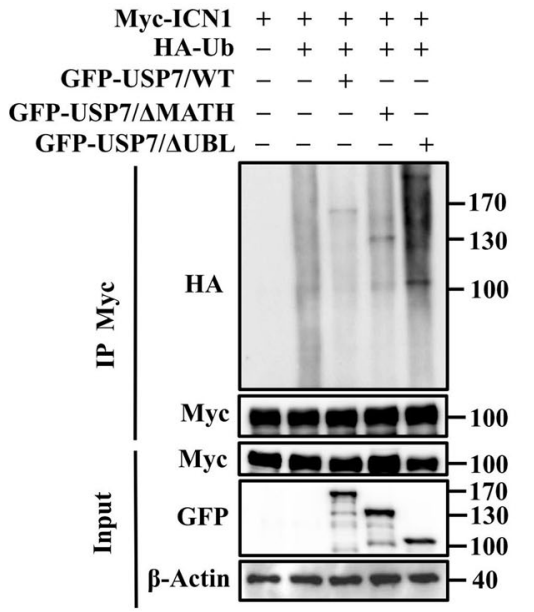

Fig. 3 USP7 deubiquitinates ICN1 in vivo and in vitro. a HEK293T cells were co-transfected with the specified plasmids. Next, cellular extracts were prepared for immunoprecipitation assays with anti-Myc antibody followed by western blotting with anti-HA antibody. b HEK293T cells were co-transfected with the indicated plasmids and treated with or without P22077 $(20 \mu \mathrm{M})$ for $6 \mathrm{~h}$ before being harvested. Further, cellular extracts were prepared for immunoprecipitation assays with anti-Myc antibody followed by western blotting with anti-HA antibody. c HEK293T cells were co-transfected with the indicated plasmids. Then, cellular extracts were immunoprecipitated with anti-Myc antibody followed by western blotting with anti-HA antibody. d HEK293T cells stably expressing shRNA specifically against USP7 were co-transfected with the indicated plasmids. Cellular extracts were immunoprecipitated with anti-Myc antibody followed by western blotting with anti-HA antibody. e In vitro deubiquitination assays. HEK293T cells were co-transfected with HA-tagged ubiquitin and Myc-tagged ICN1. Ubiquitinated-ICN1 proteins were purified using an anti-Myc antibody and then incubated with purified USP7 at $37^{\circ} \mathrm{C}$ for $2 \mathrm{~h}$, followed by western blotting with anti-HA antibody

Treatment of T-ALL cell lines (JURKAT, MOLT-4, CUTLL1, CCRF-CEM, and KOPT-K1) with P22077 led to a reduction in the ICN1 protein level in a dose-dependent manner (Fig. 1d) without changing the NOTCH1 mRNA level (Supplementary Fig. S1A and S1B). On the other hand, the proteasome-specific inhibitor MG132 blocked the P22077-induced ICN1 downregulation in JURKAT and MOLT-4 cells (Fig. 1e). These results indicate that the deubiquitinase USP7 controls the stability of NOTCH1.

USP7 interacts with ICN1

Next, we explored whether USP7 interacts with ICN1 using an immunoprecipitation assay and found that ectopically expressed Myc-ICN1 interacted with FLAG-USP7 (Fig. 2a). An interaction between endogenous USP7 and ICN1 was also validated in JURKAT and MOLT-4 cells (Fig. 2b). To investigate whether USP7 interacts directly with ICN1, we carried out GST pull-down assays. As shown in Fig. 2c, purified GST-USP7 but not GST alone could bind to FLAG-ICN1, indicating a direct interaction between USP7 and ICN1. Furthermore, the results of an immunofluorescence assay showed that USP7 and NOTCH1 were co-localized in the nucleus (Fig. 2d).

To determine the precise region of USP7 essential for its interaction with ICN1, we generated several truncated mutants of USP7 (Fig. 2e, top panel). The USP7 protein contains an N-terminal meprin, TRAF homology (MATH) domain (aa 1-208); a single catalytic domain (aa 208-560); and five C-terminal ubiquitin-like (UBL) domains (aa 560-1102). The results revealed that USP7 interacts with ICN1 through its N-terminal MATH domain and Cterminal UBL domains (Fig. 2e, bottom panel), and the UBL (1-2) domain was sufficient to bind ICN1. Nevertheless, the catalytic domain of USP7 itself could not bind ICN1 (Fig. 2e, bottom panel). These data demonstrate that USP7 interacts with ICN1 in vivo and in vitro and the MATH domain and UBL domains of USP7 mediate this interaction.

USP7 deubiquitinates ICN1

Further, we sought to determine whether USP7, as a deubiquitinase, catalyzes the deubiquitination of ICN1. For this purpose, we prepared HEK293T cells, which were transfected with expression plasmids encoding HA-ubiquitin and Myc-ICN1 with or without $\mathrm{USP}^{\mathrm{WT}}$ or USP7 ${ }^{\mathrm{C} 223 \mathrm{~S}}$. Ubiquitinated-ICN1 was immunoprecipitated with an anti-Myc antibody and detected by western blotting with anti-HA antibody. As expected, co-expression of ICN1 with wild-type USP7 but not the catalytically inactive USP7 ${ }^{C 223 S}$ decreased the protein levels of ubiquitinated ICN1 (Fig. 3a). Consistent with this finding, inhibition of USP7 by P22077 completely blocked the ability of USP7 to remove ubiquitin from ICN1 (Fig. 3b). Of note, USP7 mutants lacking the MATH domain or 


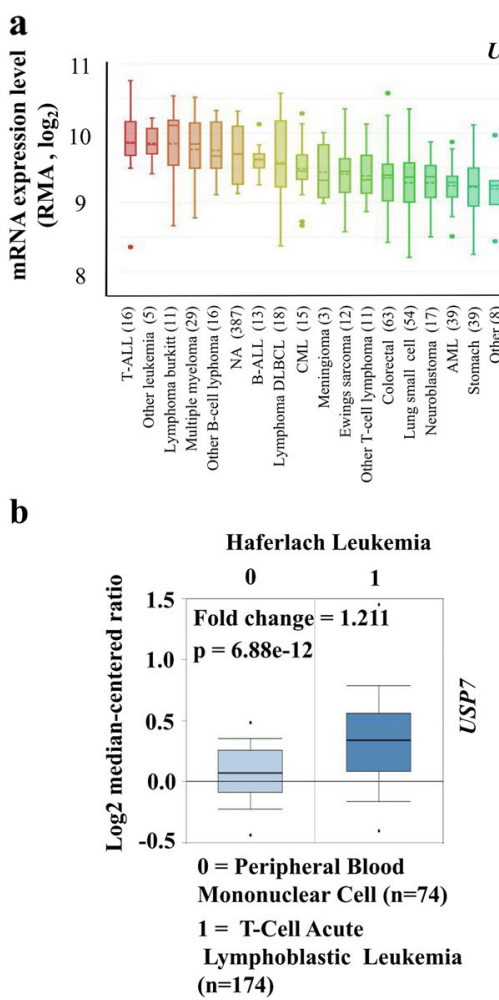

USP7

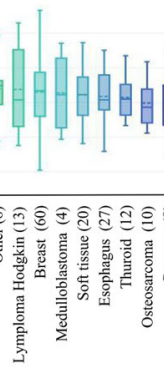

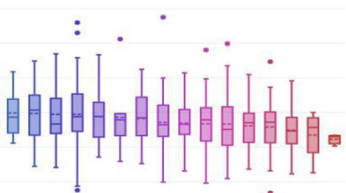

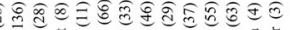
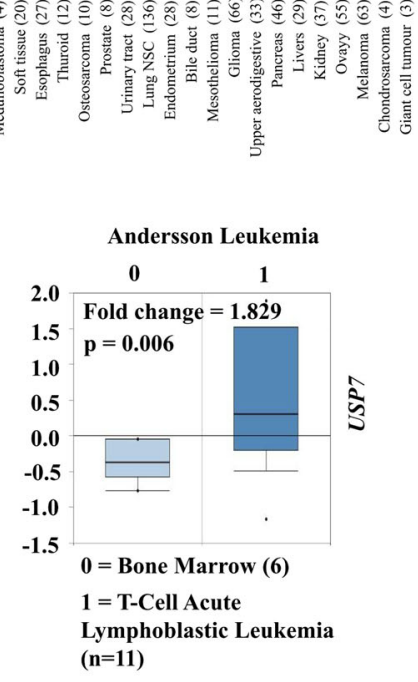

d

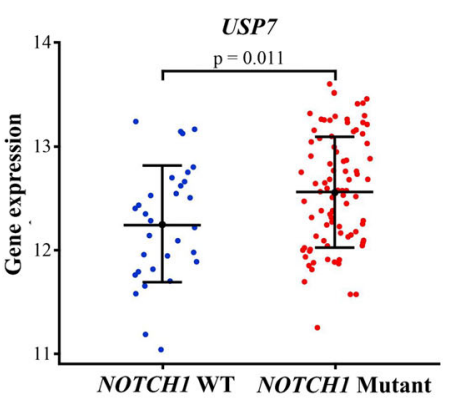

Normal T-ALL patient

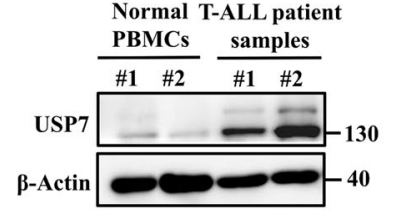

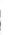

Fig. 4 USP7 is overexpressed in T-ALL. a USP7 microarray gene expression data were obtained from the Cancer Cell Line Encyclopedia (CCLE). The data are presented with box plots. The sample number $(n)$ are indicated in parentheses. RMA represents Robust Multi-array Average. $\mathbf{b}$ Analysis of TCGA leukemia dataset from the Oncomine database to assess the expression of USP7 in normal bone marrow or peripheral blood cells and in T-ALL patient samples. The data are presented with box plots. Fold change, $p$-value (determined by Student's $t$-test), and sample size are shown. c Comparison of USP7 gene expression levels between NOTCH1 WT $(n=31)$ and NOTCH1 mutated ( $n=87)$ T-ALL cases. The read counts mapped to the USP7 transcript were normalized and applied with variance-stabilizing transformation. The $p$-value was determined using Student's $t$-test. Dots represent the value of the USP7 expression level in each of the T-ALL cases. The mean and 25th and 75th percentiles are represented by the midline and line edges in the plots, respectively. $\mathbf{d}$ western blotting analysis of the USP7 protein levels in normal PBMCs and T-ALL patient samples (top panel) along with various T-ALL cell lines (bottom panel)

UBL domains could not remove ubiquitin from ICN1 (Fig. 3c), confirming the essential role of the MATH domain and UBL domains in mediating the deubiquitination activity of USP7 for ICN1. Moreover, HEK293T cells stably expressing shRNA specifically against USP7 were co-transfected with plasmids encoding HA-ubiquitin and Myc-ICN1. The protein levels of ubiquitinated ICN1 species increased after the knockdown of USP7 (Fig. 3d). To further confirm our hypothesis that USP7 is a direct DUB for ICN1, we performed an in vitro deubiquitination assay. Ubiquitinated ICN1 proteins were purified from HEK293T cells and incubated with commercially available purified USP7 protein. As illustrated in Fig. 3e, the purified USP7 markedly reduced the ubiquitination of ICN1. Taken together, the results of these experiments indicate that USP7 targets ICN1 for deubiquitination, supporting the proposal that ICN1 is a direct substrate for the deubiquitinase USP7.

USP7 is overexpressed in T-ALL

To assess the USP7 expression in T-ALL, we first analyzed the transcript expression in a wide array of human cancer cell lines and discovered that USP7 was highly expressed in T-ALL (Fig. 4a). To extend this observation, we carried out bioinformatics analysis using TCGA leukemia datasets from the public database Oncomine. USP7 was markedly upregulated in T-ALL samples compared with its expression in normal bone marrow or peripheral blood cells (Fig. 4b). Interestingly, the expression level of USP7 was considerably higher in patients suffering from NOTCH1 mutant T-ALL than in those with wild-type NOTCH1 (Fig. 4c). Additionally, USP7 was highly expressed in both human
T-ALL cell lines (Fig. 4d, bottom panel) and primary T-ALL patient samples ( $n=2$; Fig. $4 \mathrm{~d}$, top panel). Overall, these observations indicate that USP7 tends to be expressed at a higher level in human T-ALL.

Knockdown of USP7 reduces ICN1 protein levels and suppresses TALL cell proliferation in vitro and in vivo

Since NOTCH1 plays a significant role in T-ALL pathogenesis, we proposed that loss of function of USP7, which deubiquitinates and stabilizes ICN1, might inhibit T-ALL cell growth. As depicted in Fig. $5 \mathrm{a}, \mathrm{b}$ (left panel), knockdown of USP7 by two individual shRNAs significantly reduced the endogenous ICN1 protein levels in JURKAT and MOLT-4 cells without affecting the NOTCH1 mRNA level (Fig. 5a, b, right panel). Western blotting analysis revealed that USP7 depletion was associated with a decreased ICN1 half-life (Fig. 5c). Moreover, the knockdown of USP7 significantly suppressed the proliferation of JURKAT and MOLT-4 cells (Fig. $5 \mathrm{~d}$ ).

To further confirm these observations in vivo, 2.5 million JURKAT cells with or without USP7 expression were intravenously injected into NOD/SCID/IL2Ry-null (NSG) mice. At 23 days postengraftment, the control cohort showed typical leukemia phenotypes and began to die. Notably, the spleens of the USP7-depleted cohort were much smaller than those of the control cohort (Fig. 5e). Hematoxylin and eosin (H\&E) staining indicated that the USP7-depleted mice had less infiltration of lymphoblastic leukemia cells into the spleen in comparison with the control mice (Fig. 5f). Moreover, Wright's staining analysis revealed massive elevation of the number of human leukemia cells in the peripheral blood and bone marrow from the control mice. In contrast, much 

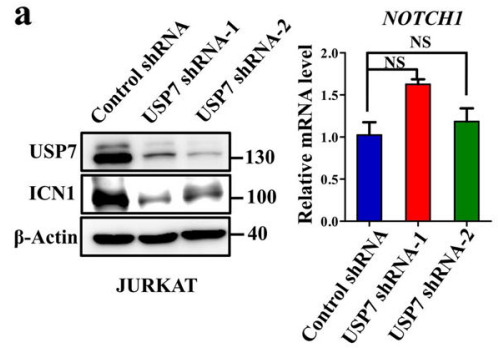

b

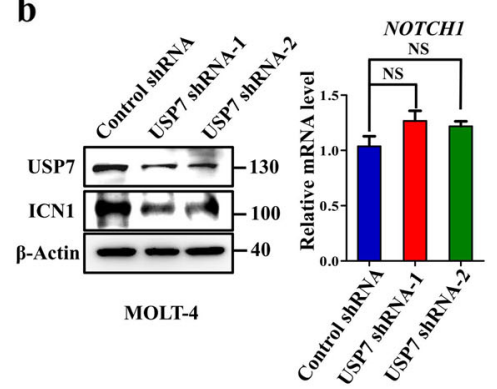

c

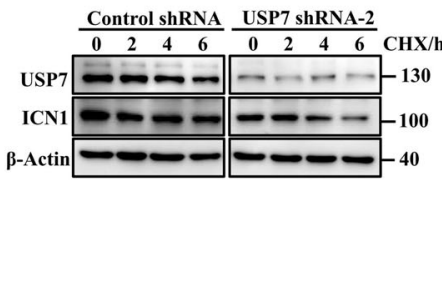

d

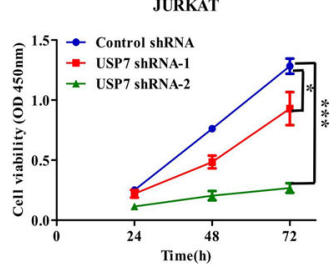

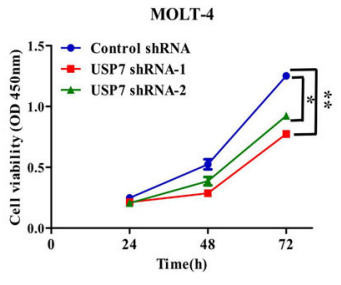

e

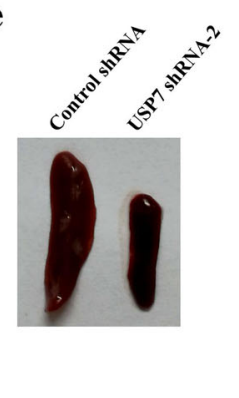

f

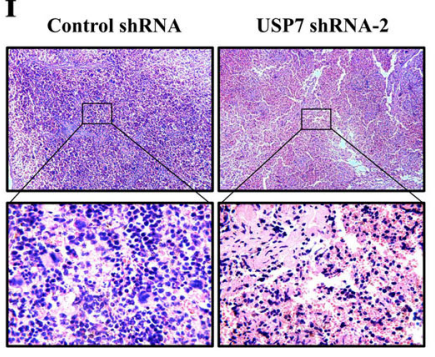

g

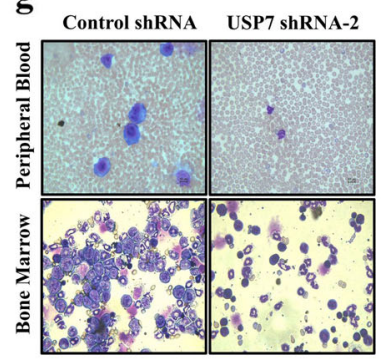

Fig. 5 Knockdown of USP7 reduces ICN1 protein levels and suppresses T-ALL cell proliferation in vitro and in vivo. $\mathbf{a}$, $\mathbf{b}$ Knockdown of USP7 reduces ICN1 protein levels. JURKAT (a) and MOLT-4 cells (b) were infected with control shRNA or two different shRNAs against USP7. The protein expression levels of USP7 and ICN1 were detected using western blotting (left panel), and the mRNA levels were determined by qRTPCR (right panel). The data are presented as the mean \pm S.D. (Student's $t$-test) of three independent experiments; NS denotes no significant. c JURKAT cells transfected with control shRNA or USP7 shRNAs were treated with $\mathrm{CHX}(50 \mu \mathrm{g} / \mathrm{mL})$ and harvested at the indicated time points, followed by western blotting analysis. $\mathbf{d}$ JURKAT and MOLT-4 cells were infected with control shRNA or USP7 shRNAs, and cell proliferation was monitored by a CCK-8 assay. The data are presented as the mean \pm S.D. (Student's $t$-test) of three independent experiments; ${ }^{*} p<0.05,{ }^{* *} p<$ 0.01 , and ${ }^{* * *} p<0.001$. e JURKAT cells stably expressing the control shRNA or USP7 shRNAs were intravenously injected into NSG mice ( $n=3$ per group). After 3 weeks, the mice were killed, and their T-ALL progression was analyzed. Representative spleen images are shown. $f$ Representative spleen H\&E staining is presented (top, 200x; bottom, 1000x). g Wright's staining of peripheral blood (top panel) and bone marrow (bottom panel) cytospin samples from the control mice and USP7-depleted mice

fewer human leukemia cells were detected in the USP7-depleted mice (Fig. $5 \mathrm{~g}$ ). These studies provide compelling evidence supporting the critical importance of USP7 as an upstream mediator of NOTCH1 in the regulation of T-ALL proliferation.

Pharmacologic inhibition of USP7 induces apoptosis in T-ALL cells Encouraged by the observation that the knockdown of USP7 inhibited the proliferation of T-ALL cells, we next evaluated the effect of the USP7 inhibitor P22077 on cell viability in four T-ALL cell lines. The results showed that P22077 decreased the cell viability in a dose-dependent manner (Fig. 6a). More importantly, P22077 induced a dose-dependent cytotoxicity in primary T-ALL cells isolated from PBMCs of two T-ALL patients (Fig. 6b). Using an Annexin V/PI double-staining assay, we discovered that P22077 induced a remarkable level of apoptosis in CCRF-CEM and CUTLL1 cells (Fig. 6c). We also tested the anti-tumor effect of P22077 on other B-cell-derived hematologic malignant cells and found that T-ALL cells exhibited high sensitivity to P22077 treatment (Supplementary Fig. S2). These results indicate that USP7 inhibition by P22077 is cytotoxic to T-ALL cell lines and patient T-ALL cells.

\section{DISCUSSION}

The NOTCH1 signaling pathway enables direct transduction of extracellular signals at the cell membrane into transcriptional responses in the nucleus and plays a critical role during $T$ cell development. It is also involved in the pathogenesis of over $60 \%$ of T-ALL cases. ${ }^{6}$ Accumulating evidence suggests that controlling the stability of NOTCH1 represents a novel approach to regulate the NOTCH1 signaling pathway and thus is attracting increasingly more attention. In the present study, for the first time, we identified USP7 as the deubiquitinase that maintains
NOTCH1 stability and protects NOTCH1 from proteasomemediated degradation. Therefore, targeting USP7 may represent a potential novel strategy to combat T-ALL.

The identification of frequent activating NOTCH1 mutations in TALL has attracted significant interest in targeting the NOTCH1 signaling pathway for treatment. ${ }^{11}$ Several strategies have been undertaken to block NOTCH1 pathway signal transduction, including preventing ligand-triggered activation or NOTCH1 autoactivation by using monoclonal antibodies such as OMP-52M51, which binds to the negative regulatory domain of NOTCH $1 .{ }^{43}$ In addition, $\gamma$-secretase inhibitors (GSIs) have been utilized to inhibit the $\gamma$-secretase-induced cleavage and release of the intracellular domain of NOTCH1 (ICN1). GSIs are the most studied potential agents targeting the NOTCH1 pathway. However, in previous studies, these inhibitors showed limited efficacy and intolerable side-effects. ${ }^{7}$ Recent studies revealed that proteasome inhibitors exert cytotoxicity and increase the chemosensitivity of T-ALL cells through suppression of NOTCH1 transcription. ${ }^{44}$ In addition, activation of the E3 ubiquitin ligase CHIP results in degradation of NOTCH1 and inhibits the proliferation of T-ALL cells. ${ }^{15}$ Based on the findings of these studies, we hypothesized that the inhibition of NOTCH1 deubiquitination catalysis processes might promote degradation of NOTCH1 and block the oncogenic NOTCH1 pathway. Here, we established that USP7 is responsible for NOTCH1 stabilization, which was supported by several lines of evidence. First, our results showed that USP7 interacted and was co-localized with ICN1 in TALL cells. Second, overexpression of USP7 removed ubiquitin from ICN1 in vivo and directly eliminated ubiquitin from purified ubiquitinated ICN1 in vitro. In contrast, USP7 inhibition increased ICN1 ubiquitination. Similar to its interaction with MDM2 or $\mathrm{p} 53{ }^{24}$ both the $\mathrm{N}$-terminal MATH domain and the C-terminal UBL domains of USP7 are needed for interaction with ICN1. The 


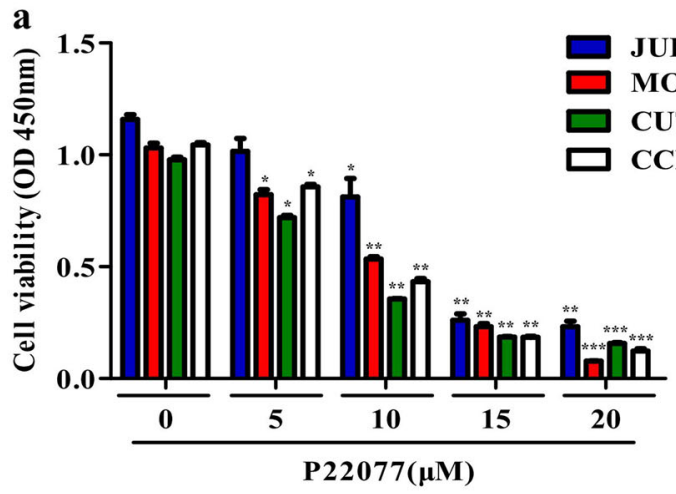

c

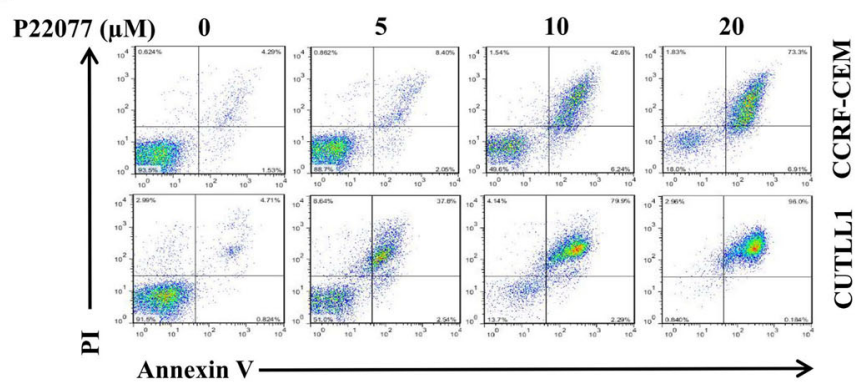

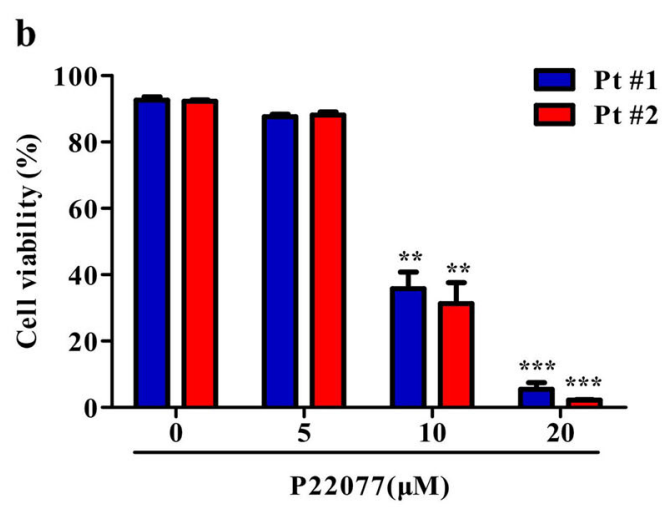

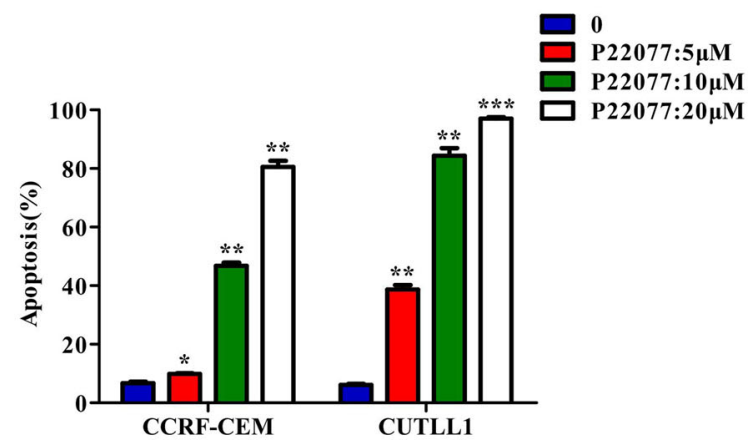

Fig. 6 USP7 inhibition induces apoptosis in T-ALL cells. a T-ALL cell lines (JURKAT, MOLT-4, CUTLL1, and CCRF-CEM) were treated with increasing concentrations of P22077 for $24 \mathrm{~h}$, followed by assessment of cell viability using a CCK-8 assay. The data are presented as the mean \pm S.D. (Student's $t$-test) of three independent experiments; ${ }^{*} p<0.05,{ }^{* *} p<0.01$, and ${ }^{* * *} p<0.001$. b Primary T-ALL cells were isolated and treated with different concentrations of P22077 for $24 \mathrm{~h}$, and the cell viability was monitored via trypan blue staining. The data are presented as the mean \pm S.D. (Student's $t$-test); ${ }^{* *} p<0.01$, and ${ }^{* * *} p<0.001$. c CCRF-CEM and CUTLL1 cells were treated with various concentrations of P22077 for $48 \mathrm{~h}$. Cell apoptosis was determined via flow cytometry using an Annexin-V/PI dual staining assay. Columns (right panel) represent the average percentage of Annexin V-positive cells from three independent experiments, shown as the mean \pm S.D. (Student's $t$-test); ${ }^{*} p<0.05,{ }^{* *} p$ $<0.01$, and ${ }^{* * *} p<0.001$

absence of either domain reverses USP7-mediated deubiquitination of ICN1. It is likely that ICN1 competes with MDM2 or p53 to bind USP7 in T-ALL. Finally, inhibition of the USP7 catalytic activity or depletion of USP7 reduced the protein level of ICN1 without affecting the mRNA level. Interestingly, USP7 inhibition caused degradation of ICN1 in T-ALL cell lines regardless of the status of NOTCH1 (JURKAT and CCRF-CEM cells have FBW7 mutations; MOLT-4 and KOPT-K1 cells have PEST domain mutations; ${ }^{45}$ CUTLL1 cells harbor a chromosomal translocation at $(7 ; 9)(q 34$; $\left.\mathrm{q} 34)^{46}\right)$. These findings indicate that other E3 ligases, such as CHIP, may be involved in the NOTCH1 ubiquitination catalysis process in T-ALLs with FBW7 mutations or PEST domain mutations. Thus, these observations provide new insight into the regulation of the NOTCH1 deubiquitination processes in T-ALL cells.

USP7 has been found to be a drug target in several cancer types. However, its role in T-ALL is unclear. Similar to other cancers, USP7 is overexpressed in T-ALL, suggesting a possible role in T-ALL pathogenesis. In this regard, we discovered that knockdown of USP7 blocked T-ALL cell proliferation in vitro and in vivo, and inhibition of USP7 resulted in T-ALL cell growth suppression and apoptosis. Interestingly, USP7 inhibitor suppressed cell growth in both GSI-sensitive (CUTLL1) and GSIresistant (JURKAT, MOLT-4, and CCRF-CEM) T-ALL cells, thus providing a rationale for inclusion of USP7 inhibitors in a GSIbased regimens for treatment of T-ALL patients. More importantly, we found that USP7 is overexpressed in human T-ALL, which likely results from the constitutive activation of NOTCH1. Bioinformatics analysis revealed that the USP7 mRNA expression was higher in NOTCH1 mutant than in the NOTCH1 wild-type T-ALL patients, indicating that mutation of NOTCH1 may upregulate the mRNA level of USP7. A positive feedback loop may exist between USP7 and NOTCH1: USP7 stabilizes and deubiquitinates NOTCH1, which in turn facilitates transactivation of USP7. Further studies are needed to verify this speculation.

Although the induction of $\mathrm{NOTCH} 1$ degradation may play a major role, we could not rule out the possibility that other USP7 substrates also contribute to the anti-leukemia effects upon USP7 inhibition. For example, PTEN has been shown to be involved in the pathogenesis of T-ALL. ${ }^{47}$ In an earlier study, USP7 removed PTEN monoubiquitination, enhanced its nuclear export, and abolished its nuclear function. ${ }^{21}$ MDM2 is also a substrate of USP7, and inhibition of USP7 resulted in degradation of MDM2. Nutlin-3a, an antagonist of MDM2, was reported to be able induce apoptosis in T-ALL cells with wild-type p53. ${ }^{48}$ These reports further support the notion that targeting USP7 is helpful in the treatment of T-ALL.

One concern for targeting USP7 in T-ALL emerges from recent observations of frequent loss-of-function mutations in USP7 found in pediatric leukemia, especially in TAL1/LMO1-positive T-ALL cases. ${ }^{38,49,50}$ The role of USP7 in these patients remains enigmatic. Possibly, USP7 may function as a tumor suppressor. However, we cannot rule out the likelihood that USP7 may exert other catalyticindependent roles in these patients. These observations add to the complexity of the potential application of USP7-based therapy in T-ALL patients. Future studies are required to characterize the patients suitable for USP7-targeting therapy.

In summary, we identified USP7 as a novel deubiquitinase for NOTCH1 and found that targeting USP7 promoted NOTCH1 degradation, thus providing a novel means to terminate NOTCH1 signaling. Our findings provide a molecular basis and rationale for inclusion of USP7 inhibitors in T-ALL treatment strategies. 


\section{ACKNOWLEDGEMENTS}

We would like to express our gratitude to Prof. Hudan Liu for providing the ICN1expression plasmids. We are also thankful to Prof. Jing Liu for the kind gift of various USP7 constructs. This work was supported in part by grants from the National Key Research and Development Program of China (no. 2017YFA0505200), the National Basic Research Program of China (973 Program) (no. 2015CB910403), the National Natural Science Foundation of China (81700475, 81670139, 81570118, and 81570112), Natural Science Foundation of Shanghai (16ZR1427800), the Science and Technology Committee of Shanghai (15401901800), and the Innovation Program of Shanghai Municipal Education Commission (13YZ028)

\section{AUTHOR CONTRIBUTIONS}

H.S. and Y.W. conceived and designed the study; H.S., X.L., X.X., J.S., M.L., H.L., and H.X. performed the experiments; H.S., Y.W., Y.D. and J.H. analyzed data; L.Y., Y.T., and L.Z. provided reagents and/or technical support; H.S., Y.W., and X.L. wrote and revised the manuscript. All authors read and approved the final version.

\section{ADDITIONAL INFORMATION}

The online version of this article (https://doi.org/10.1038/s41392-018-0028-3) contains supplementary material, which is available to authorized users.

Competing interests: The authors declare no competing interests.

\section{REFERENCES}

1. Yamamoto, S., Schulze, K. L. \& Bellen, H. J. Introduction to Notch signaling. Methods Mol. Biol. 1187, 1-14 (2014).

2. Rizzo, P. et al. Rational targeting of Notch signaling in cancer. Oncogene 27, 5124-5131 (2008).

3. Weng, A. P. et al. c-Myc is an important direct target of Notch1 in T-cell acute lymphoblastic leukemia/lymphoma. Genes Dev. 20, 2096-2109 (2006).

4. Kishi, N. et al. Murine homologs of deltex define a novel gene family involved in vertebrate Notch signaling and neurogenesis. Int. J. Dev. Neurosci. 19, 21-35 (2001).

5. Wendorff, A. A. et al. Hes1 is a critical but context-dependent mediator of canonical Notch signaling in lymphocyte development and transformation. Immunity 33, 671-684 (2010).

6. Weng, A. P. et al. Activating mutations of NOTCH1 in human T cell acute lymphoblastic leukemia. Science 306, 269-271 (2004).

7. Paganin, M. \& Ferrando, A. Molecular pathogenesis and targeted therapies for NOTCH1-induced T-cell acute lymphoblastic leukemia. Blood. Rev. 25, 83-90 (2011).

8. Gao, L., Yuan, K., Ding, W. \& Lin, M. Notch signaling: a potential therapeutic target for hematologic malignancies. Crit. Rev. Eukaryot. Gene Expr. 26, 239-246 (2016).

9. Girardi, T., Vicente, C., Cools, J. \& De Keersmaecker, K. The genetics and molecular biology of T-ALL. Blood 129, 1113-1123 (2017).

10. Katsuya, H. \& Ishitsuka, K. Treatment advances and prognosis for patients with adult T-cell leukemia-lymphoma. J. Clin. Exp. Hematop. 57, 87-97 (2017).

11. Durinck, K. et al. Novel biological insights in T-cell acute lymphoblastic leukemia. Exp. Hematol. 43, 625-639 (2015).

12. Vilimas, T. et al. Targeting the NF-kappaB signaling pathway in Notch1-induced Tcell leukemia. Nat. Med. 13, 70-77 (2007).

13. Chan, S. M., Weng, A. P., Tibshirani, R., Aster, J. C. \& Utz, P. J. Notch signals positively regulate activity of the mTOR pathway in T-cell acute lymphoblastic leukemia. Blood 110, 278-286 (2007).

14. Mo, J. S. et al. Integrin-linked kinase controls Notch1 signaling by downregulation of protein stability through Fbw7 ubiquitin ligase. Mol. Cell. Biol. 27, 5565-5574 (2007).

15. Wang, Z. et al. Stabilization of notch1 by the Hsp90 chaperone is crucial for T-cell leukemogenesis. Clin. Cancer Res. 23, 3834-3846 (2017).

16. Jehn, B. M., Dittert, I., Beyer, S., von der Mark, K. \& Bielke, W. c-Cbl binding and ubiquitin-dependent lysosomal degradation of membrane-associated Notch1. J. Biol. Chem. 277, 8033-8040 (2002).

17. Komander, D., Clague, M. J. \& Urbe, S. Breaking the chains: structure and function of the deubiquitinases. Nat. Rev. Mol. Cell Biol. 10, 550-563 (2009).

18. Moretti, J. et al. The translation initiation factor $3 \mathrm{f}$ (elF3f) exhibits a deubiquitinase activity regulating Notch activation. PLoS Biol. 8, e1000545 (2010).

19. Bhattacharya, S., Chakraborty, D., Basu, M. \& Ghosh, M. K. Emerging insights into HAUSP (USP7) in physiology, cancer and other diseases. Signal Transduct. Target Ther. 3, 17 (2018).

20. van der Horst, A. et al. FOXO4 transcriptional activity is regulated by monoubiquitination and USP7/HAUSP. Nat. Cell Biol. 8, 1064-1073 (2006).
21. Song, M. S. et al. The deubiquitinylation and localization of PTEN are regulated by a HAUSP-PML network. Nature 455, 813-817 (2008).

22. Morotti, A. et al. Unleashing the guardian: the targetable BCR-ABL/HAUSP/PML/ PTEN network in chronic myeloid leukemia. Curr. Drug Targets 18, 389-395 (2017).

23. Li, M. et al. Deubiquitination of $\mathrm{p} 53$ by HAUSP is an important pathway for p53 stabilization. Nature 416, 648-653 (2002).

24. $\mathrm{Ma}$, J. et al. C-terminal region of USP7/HAUSP is critical for deubiquitination activity and contains a second mdm2/p53 binding site. Arch. Biochem. Biophys. 503, 207-212 (2010).

25. Tavana, O. et al. HAUSP deubiquitinates and stabilizes N-Myc in neuroblastoma. Nat. Med. 22, 1180-1186 (2016).

26. Felle, M. et al. The USP7/Dnmt1 complex stimulates the DNA methylation activity of Dnmt 1 and regulates the stability of UHRF1. Nucleic Acids Res. 39, 8355-8365 (2011).

27. Qin, W., Leonhardt, H. \& Spada, F. Usp7 and Uhrf1 control ubiquitination and stability of the maintenance DNA methyltransferase Dnmt1. J. Cell. Biochem. 112 439-444 (2011).

28. Inoue, D., Nishimura, K., Kozuka-Hata, H., Oyama, M. \& Kitamura, T. The stability of epigenetic factor ASXL1 is regulated through ubiquitination and USP7-mediated deubiquitination. Leukemia 29, 2257-2260 (2015).

29. Wang, Q. et al. Stabilization of histone demethylase PHF8 by USP7 promotes breast carcinogenesis. J. Clin. Invest. 126, 2205-2220 (2016).

30. van Loosdregt, J. et al. Stabilization of the transcription factor Foxp3 by the deubiquitinase USP7 increases Treg-cell-suppressive capacity. Immunity 39, 259-271 (2013).

31. Colland, F. et al. Small-molecule inhibitor of USP7/HAUSP ubiquitin protease stabilizes and activates p53 in cells. Mol. Cancer Ther. 8, 2286-2295 (2009).

32. Chauhan, D. et al. A small molecule inhibitor of ubiquitin-specific protease-7 induces apoptosis in multiple myeloma cells and overcomes bortezomib resistance. Cancer Cell. 22, 345-358 (2012).

33. Turnbull, A. P. et al. Molecular basis of USP7 inhibition by selective smallmolecule inhibitors. Nature 550, 481-486 (2017).

34. Jing, B. et al. Characterization of naturally occurring pentacyclic triterpenes as novel inhibitors of deubiquitinating protease USP7 with anticancer activity in vitro. Acta Pharmacol. Sin. 39, 492-498 (2018).

35. Qin, D. et al. CDDO-Me reveals USP7 as a novel target in ovarian cancer cells. Oncotarget 7, 77096-77109 (2016).

36. Barretina, J. et al. The Cancer Cell Line Encyclopedia enables predictive modelling of anticancer drug sensitivity. Nature 483, 603-607 (2012).

37. Rhodes, D. R. et al. Oncomine 3.0: genes, pathways, and networks in a collection of 18,000 cancer gene expression profiles. Neoplasia 9, 166-180 (2007).

38. Chen, B. et al. Identification of fusion genes and characterization of transcriptome features in T-cell acute lymphoblastic leukemia. Proc. Natl Acad. Sci. USA 115, 373-378 (2018)

39. Dobin, A. et al. STAR: ultrafast universal RNA-seq aligner. Bioinformatics 29, 15-21 (2013).

40. Anders, S., Pyl, P. T. \& Huber, W. HTSeq--a Python framework to work with highthroughput sequencing data. Bioinformatics 31, 166-169 (2015).

41. Love, M. I., Huber, W. \& Anders, S. Moderated estimation of fold change and dispersion for RNA-seq data with DESeq2. Genome Biol. 15, 550 (2014).

42. Weinstock, J. et al. Selective dual inhibitors of the cancer-related deubiquitylating proteases USP7 and USP47. ACS Med. Chem. Lett. 3, 789-792 (2012).

43. Agnusdei, V. et al. Therapeutic antibody targeting of Notch1 in T-acute lymphoblastic leukemia xenografts. Leukemia 28, 278-288 (2014).

44. Koyama, D. et al. Proteasome inhibitors exert cytotoxicity and increase chemosensitivity via transcriptional repression of Notch1 in T-cell acute lymphoblastic leukemia. Leukemia 28, 1216-1226 (2014).

45. O'Neil, J. et al. FBW7 mutations in leukemic cells mediate NOTCH pathway activation and resistance to gamma-secretase inhibitors. J. Exp. Med. 204, 1813-1824 (2007).

46. Palomero, T. et al. CUTLL1, a novel human T-cell lymphoma cell line with $t(7 ; 9)$ rearrangement, aberrant NOTCH1 activation and high sensitivity to gammasecretase inhibitors. Leukemia 20, 1279-1287 (2006).

47. Palomero, T. et al. Mutational loss of PTEN induces resistance to NOTCH1 inhibition in T-cell leukemia. Nat. Med. 13, 1203-1210 (2007).

48. Hasegawa, $\mathrm{H}$. et al. Activation of $\mathrm{p} 53$ by Nutlin-3a, an antagonist of MDM2, induces apoptosis and cellular senescence in adult T-cell leukemia cells. Leukemia 23, 2090-2101 (2009).

49. Huether, R. et al. The landscape of somatic mutations in epigenetic regulators across 1,000 paediatric cancer genomes. Nat. Commun. 5, 3630 (2014).

50. Liu, Y. et al. The genomic landscape of pediatric and young adult T-lineage acute lymphoblastic leukemia. Nat. Genet. 49, 1211-1218 (2017). 
Open Access This article is licensed under a Creative Commons Attribution 4.0 International License, which permits use, sharing, adaptation, distribution and reproduction in any medium or format, as long as you give appropriate credit to the original author(s) and the source, provide a link to the Creative Commons license, and indicate if changes were made. The images or other third party material in this article are included in the article's Creative Commons license, unless indicated otherwise in a credit line to the material. If material is not included in the article's Creative Commons license and your intended use is not permitted by statutory regulation or exceeds the permitted use, you will need to obtain permission directly from the copyright holder. To view a copy of this license, visit http://creativecommons. org/licenses/by/4.0/.

(c) The Author(s) 2018 